\title{
Cartografías de la desigualdad: una década de conflictos de vivienda y nuevas resistencias en Santiago de Chile. Análisis del conflicto de la Maestranza de San Eugenio
}

Luis del Romero. Universidad de Valencia, Valencia, España.

RESUMEN | La vivienda es en todo el mundo uno de los motivos de conflicto social más importantes en cualquier ciudad. En el caso chileno y con el movimiento de pobladores, los conflictos por la vivienda han cobrado además un protagonismo social y político muy relevante. El objetivo de este trabajo es, en primer lugar, realizar una actualización de la geografía de los conflictos de vivienda en Santiago de Chile; y en segundo lugar, analizar cómo el repertorio de protesta y las narrativas de los movimientos de pobladores han innovado y evolucionado notablemente desde su apogeo de los años sesenta hasta 1973, aumentando aun si cabe su complejidad, como veremos con un caso de conflicto: el proyecto de vivienda social de Ukamau en el predio de la Maestranza de San Eugenio.

PALABRAS ClaVe | conflicto social, vivienda, movimientos sociales.

ABSTRACT | Worldwide, housing is one of the most important causes for social conflict in any city; however, in the case of Chile and the pobladores movement, conflicts over housing have become a very important social and political issue. The aim of this work is to first carry out an update of the geography of the housing conflict in Santiago, Chile, and secondly to analyze how the repertoire of protest and narratives of the pobladores movement have innovated and evolved considerably since its high point during the decade of 1960 to 1973 and increasing its complexity, as we shall see in a case of conflict: The Ukamau Social Housing Project in the area of Maestranza de San Eugenio, Santiago de Chile.

KEYwORDs | social conflict, housing, social movements. 


\section{Introducción}

Revisando la literatura científica de los últimos años sobre movimientos de pobladores en Santiago de Chile, queda la sensación de que hay pocas novedades, aparte de algunos casos de estudio relacionados con el problema de la vivienda. A partir de los ańos noventa parece extinguirse poco a poco el tema, hasta desaparecer (Angelcos \& Pérez, 2017; Salazar, 2012, p. 190). La toma de terrenos en Peñalolén (comuna de clases medias al este de Santiago), en 1999, recordó por momentos las grandes movilizaciones y tomas vividas durante los ańos sesenta y setenta, hasta el golpe de Estado de 1973. Desde entonces, en muchos casos el interés académico parece haber declinado considerablemente y no estar ya en la agenda de investigación en cuestiones sociales en Chile, pese al reconocido carácter de discontinuidad que ha presentado históricamente el movimiento de pobladores (Cortés, 2014, p. 246). Esto constituye una paradoja, ya que los pobladores fueron un actor clave en la lucha por la recuperación de la democracia y un movimiento social de referencia en el Chile urbano, pero desde los años noventa parecen haber sido excluidos del debate público, especialmente el académico (Angelcos \& Pérez, 2017; Oxhorn, 2004; Salazar, 2012).

En los últimos años, sin embargo, ha habido cambios sustanciales tanto en las políticas urbanas y de vivienda del Estado, como en las estrategias de organización y resistencia de los movimientos de pobladores. Tales cambios requieren de una reflexión pausada en la arena académica, más allá del ruido mediático que generan episodios esporádicos de protestas de pobladores, así como del cortoplacismo cuatrienal en el que se enmarcan las políticas urbanas en Chile. En síntesis, la política habitacional en las últimas dos décadas se ha basado en la construcción masiva de vivienda social financiada por el Estado y llevada a cabo por empresas privadas en la periferia urbana (Castillo, 2011, p. 134). Esta política, sustentada en fuertes inversiones públicas, además de posibilitar una relativa reducción del déficit de viviendas (Ducci, 1997), constituye históricamente un elemento dinamizador de la economía y una fuente de pingües beneficios para el capital financiero y las empresas de construcción (Castells, 1973, p. 11), aunque con el paso de los ańos se haya mejorado algunos aspectos de las políticas de subsidio. En 2006 comenzó el gobierno de la Concertación presidido por la socialista Michelle Bachelet y que se extendió hasta 2010, y de nuevo en 2013 volvió a presidir el país. En este periodo se introdujo un subsidio complementario de localización para financiar el mayor costo de los terrenos en las comunas más caras, y aumentó el subsidio del Fondo Solidario de Vivienda (FSV) para mejorar la calidad de las viviendas (Castillo, 2011, p. 134). Además de estos ajustes, se incorporó otros programas que conformaron una nueva política habitacional, mediante los cuales se creó la figura de las Entidades de Gestión Inmobiliaria Social (EGIS). Se trata de agencias, con o sin fines de lucro, encargadas de dar asesoría especializada para guiar y respaldar a las personas que postulen a los programas del Ministerio de Vivienda y Urbanismo (Minvu).

Desde el punto de vista del movimiento de pobladores, más que un agotamiento de sus discursos o una desmovilización — lo que podría justificar el desinterés académico-, se ha producido una importante reestructuración del movimiento para 
constituir un actor social participativo en la producción de espacio y sociedad, de manera que sus métodos de acción, así como sus objetivos de lucha, se han ampliado y diversificado (Angelcos, 2015; Salazar, 2012, p. 190). En julio de 2006, nació en la comuna de Peñalolén el Movimiento de Pobladores en Lucha (MPL) (Guzmán, Renna, Sandoval \& Silva, 2009, p. 4), un nuevo actor que renovó profundamente el panorama de los movimientos de pobladores existente hasta ese momento, tanto desde el punto de vista discursivo como organizativo, más allá de las clásicas tomas de terrenos, como veremos más adelante. Cuatro años más tarde, inmediatamente después del gran terremoto de 2010, nació la Federación Nacional de Pobladores (Fenapo), que agrupa a diversos movimientos de diferentes ciudades del país. Por último, un año más tarde, se creó Ukamau, uno de los movimientos de pobladores más activos de los últimos años en la capital chilena. Así, mientras que en años recientes se han dado pasos políticos importantes para instaurar políticas de vivienda más inclusivas, no solo han surgido nuevos movimientos de pobladores, sino que el conflicto por la vivienda en Santiago de Chile se ha manifestado intensamente en numerosas comunas pericentrales de la Región Metropolitana de Santiago, lo que evidencia que estas políticas desplegadas desde los gobiernos de centro izquierda han recibido una fuerte contestación social. Por lo tanto, hay bastante que examinar y analizar. El objetivo de este trabajo es, en primer lugar, realizar una actualización de la geografía de los conflictos de vivienda en Santiago de Chile; y, en segundo lugar, analizar cómo el repertorio de protesta y las narrativas de los movimientos de pobladores han innovado y evolucionado notablemente desde su apogeo de los ańos sesenta hasta 1973, aumentando su complejidad, como veremos con un caso de conflicto: el proyecto de vivienda social de Ukamau en el predio de la Maestranza de San Eugenio.

El primer objetivo se aborda utilizando como base los trabajos previos de la Corporación sur en la elaboración de un mapa de los conflictos territoriales que se dieron en Santiago de Chile entre 2006 y 2011 (sur, 2011). Además, se complementa con noticias de conflictos del diario El Mercurio, principalmente, y Radio Universidad de Chile en menor medida, así como a través de un proyecto digital de recopilación de relatos de conflictos territoriales (Francés, 2016). Desplegaremos el segundo objetivo a partir del análisis del conflicto originado a partir de un proyecto inmobiliario propuesto para ser desarrollado en terrenos de la Maestranza de San Eugenio, protagonizado por uno de los movimientos de pobladores más activos en la segunda década del siglo en Santiago: Ukamau. De esta manera, pretendemos matizar las tesis de la desmovilización de pobladores y mostrar la enorme complejidad social y política que revisten los actuales conflictos de vivienda.

\section{Los orígenes del malestar social: la vivienda en el urbanismo neoliberal chileno}

Afirman Peck, Theodore y Brenner (2013) que el proyecto neoliberal no es monolítico ni uniforme, sino que muchas veces se desarrolla desigualmente según cada contexto; esto es, se hibrida y se imbrica en las instituciones de gobierno de la ciudad de diferentes formas. Esto hace particularmente complejo mostrar cómo se adaptan los discursos neoliberales a la práctica y política urbana de ciudades 
concretas. La política de vivienda es, sin embargo, una de las esferas básicas donde más claramente se observa la creciente mercantilización de la ciudad y lo urbano a través de procesos de desposesión (desahucios y expropiaciones, paulatina transformación de la vivienda de derecho de ciudadanía a objeto de consumo, o la creciente importancia de su valor de cambio) y de privatización (venta de stocks de vivienda pública a fondos de inversión).

La tensión entre vivienda como derecho y como mercancía está en el origen de numerosos conflictos urbanos que han surgido en las últimas décadas, en prácticamente casi cualquier ciudad del mundo capitalista, como París, Berlín, Barcelona o Montreal (Del Romero, 2016), con resultados escasamente positivos para los actores desencadenantes de dichos conflictos (asociaciones de vecinos, pobladores, organizaciones okupas, asociaciones de defensa del patrimonio histórico, movimientos antidesahucios, etcétera). Si hay un país, sin embargo, donde el proyecto neoliberal se ha desarrollado más ampliamente y donde el conflicto por la vivienda ha alcanzado mayores cotas de tensión, es Chile. Desde el golpe de Estado apoyado por EE.UU. en 1973, fue uno de los primeros países en los que se ensayó la "doctrina del shock" neoliberal, con los "Chicago boys" como referentes intelectuales (Klein, 2007). Durante años, este país ha sido uno de los principales laboratorios de las políticas neoliberales de todo tipo, mientras las reacciones a estas políticas también han sido notables. Los movimientos estudiantiles, de obreros, de pobladores e indígenas, han tenido un papel muy destacado en la paulatina recuperación de la democracia y en la conquista de algunos derechos básicos de ciudadanía; el último de ellos, el abaratamiento paulatino — aunque muy lento- de las tasas universitarias.

En esta amalgama de movimientos, sin duda el movimiento de pobladores destaca dentro del estudio del problema de la vivienda, con casi un siglo de historia y algunos hitos importantes, en especial la construcción del barrio de La Victoria en Santiago de Chile. Tras décadas de lucha, con las políticas de subsidios en algunos casos se ha conseguido parcialmente un cierto "derecho a la vivienda", frente a lo cual son muchas las voces que reclaman que las movimientos sociales urbanos utilicen cauces estrictamente institucionales para continuar consolidando este derecho, desterrando el conflicto y la confrontación con el gobierno de la ciudad. En este sentido, algunos autores ya trabajan en la hipótesis de que gran parte de Latinoamérica ha pasado a una fase de "posneoliberalismo" (Yates \& Bakker, 2014), lo que exige una readaptación del panorama de movimientos sociales a esta nueva realidad: de la confrontación a la cooperación.

Sin embargo, también existen voces que critican la política de vivienda en Chile por su carácter continuista respecto de las políticas de la dictadura, al estar enmarcadas completamente en las lógicas neoliberales de producción del espacio (Trivelli, 2009). El objetivo fundamental desde la dictadura ha sido crear las mejores condiciones para que el mercado de la tierra pueda operar lo más libre posible (Trivelli, 2009 , p. 210). De hecho, algunos autores han planteado que la política de vivienda social en Chile no ha sido tal, sino tan solo una política de financiamiento para la construcción de viviendas baratas (Rodríguez \& Sugranyes, 2009, p. 303). En el caso de Santiago, la extrema liberalización de su política urbanística, bajo el supuesto erróneo de que más suelo en el mercado ayudaría a moderar el precio 
final de la vivienda (Trivelli, 2009), ha tenido tres consecuencias relevantes que caracterizan la ciudad:

a. Una acentuación incontrolable de la tendencia a la suburbanización y la formación de un periurbano difuso y de baja densidad, por las políticas de liberalización y desregulación urbanísticas que eliminaron obstáculos preexistentes para que se desplegase una lógica estrictamente capitalista en la producción y la reproducción metropolitana (De Mattos, 2009, p. 187), lo que vino acompañado de un vertiginoso aumento de las tasas de motorización en todo el país (Ibíd., p. 192).

b. Afirmación de una estructura metropolitana segregada y polarizada, donde la estratificación social tiene una perfecta lectura territorial entre guetos de ricos y de pobres, lo cual ha sido favorecido por las erradicaciones llevadas a cabo por el gobierno militar y por las políticas de vivienda social de la democracia (De Mattos, 2009, p. 196). Estas políticas, además, han generado un nuevo problema: los pobres "con techo", familias beneficiarias de la política de vivienda social que han recibido casas de muy mala calidad y con una localización marginal en la ciudad (Rodríguez \& Sugranyes, 2009, p. 308).

c. Irrupción de un conjunto de artefactos urbanos de la globalización, con gran capacidad para reestructurar el espacio metropolitano; entre ellos, los centros comerciales, centros empresariales, megaproyectos inmobiliarios, hoteles y centros de convenciones (De Mattos, 2009, p.199), formalizados en muchos casos a través de la figura de las Zonas Urbanizables con Desarrollo Condicionado (Zoduc), así como ciudades satelitales destinadas a ser desarrolladas por la iniciativa privada (Trivelli, 2009, p. 216).

Entre estas tres dinámicas territoriales, sin duda es la segunda la que más ha impactado sobre los pobladores de la Región Metropolitana de Santiago. Existe una aguda dualización y segregación espacial en este territorio, entre un área central y noreste (Providencia, Vitacura, Los Condes), con la concentración de altas rentas, frente a una corona metropolitana sur y suroeste (Estación Central, Maipú, Lo Prado), donde proliferan los asentamientos informales, los problemas de hacinamiento y allegamiento, y donde el gobierno apenas parece desplegar tímidamente una política de vivienda social, muy lejos de las demandas reales de dignificación de la vida urbana en el marco del "derecho a la ciudad". Se trata de una dualización dirigida, que proviene de la propia división de comunas establecida por la dictadura en los años ochenta, que separó drásticamente comunas ricas y pobres (Guzmán et al., 2009).

Las principales críticas que se hacen a las políticas de vivienda están dirigidas a las políticas de subsidio de la vivienda, que han provocado la expulsión de pobladores desde las comunas centrales o pericentrales de Santiago hacia las periferias, por el continuo aumento del precio de la vivienda. Este proceso es uno de los rasgos centrales en varias ciudades. Según Lepeyronnie (1999, citado por Angelcos, 2015), el urbanismo moderno tiende a resolver la confrontación entre grupos sociales mediante su separación física, transformando los conflictos de clase en segregación urbana. 
Ducci (1997, p. 24) afirma que las jubilosas familias beneficiarias que alcanzan el sueño de una vivienda propia, se encuentran repentinamente trasladadas a cualquier lugar de la periferia (donde se construyen las soluciones para los más pobres), lejos de su lugar de origen, lejos de la familia y antiguas amistades. El desplazamiento de familias es, por lo tanto, un gran trauma para muchos de sus miembros, cuya posibilidad de visitar a familiares y amigos es prácticamente nula debido a sus limitaciones económicas, con la consiguiente ruptura de los lazos familiares y los sistemas de solidaridad y confianza desarrollados entre antiguos vecinos en la lucha por la supervivencia en el campamento (Ducci, 1997, p. 12; Mathivet \& Pulgar, 2010). Según estos autores, la misma acción del Estado subsidiario ha sido quizás la mayor causante de problemas sociales en la ciudad, en especial por la creación de "guetos" de pobreza urbana de pobladores "con techo", los cuales dejaron de ser sujetos de derechos o agentes sociales, para transformarse en beneficiarios u objeto de programas sociales focalizados (Angelcos \& Pérez, 2017; Mathivet \& Pulgar, 2010). Con estas dinámicas de expulsión de la ciudad y reducción de muchos pobladores al estatus de beneficiarios sociales, se produce, según Zibechi (2008, p.76), una "desciudadanizacion". Los no ciudadanos son aquella parte de la sociedad desciudadanizada que perdió sus espacios y sus lugares en la sociedad neoliberal, que lucha por abrir sus propios espacios, en un proceso de luchas en el que se despliegan como sujetos. Sin embargo, conviene matizar esta idea de la desciudananización en el caso de Chile, considerando las diferentes formas de resistencia organizada protagonizadas por el movimiento de pobladores, los que reclaman, más que nuevas formas de ciudadanía, su reconocimiento como ciudadanos de pleno derecho en el Estado chileno.

\section{Conflicto por la vivienda y movimiento de pobladores 2006-2016}

\section{Nuevas estrategias y objetivos del movimiento de pobladores}

Las políticas de viviendas basadas en el subsidio y la construcción segregada propician el malestar urbano por la vivienda en Santiago. El relato comienza en 2006, con el gobierno progresista de la Concertación. En la madrugada del 12 de marzo de 2006, durante la primera semana de gobierno de Michelle Bachelet, hubo varios intentos fallidos de tomas de terrenos, protagonizados por el movimiento de pobladores "Comité Lucha y Vivienda” en Peñalolén. Fueron rechazados por la policía, lo que condujo, por una parte, a pensar nuevas estrategias para la organización y la lucha por lograr una vivienda digna; y por otra, a considerar que las tomas no podían ser un fin en sí mismo (Angelcos, 2015, p. 223; Guzmán et al., 2009, p. 10). En julio de ese mismo año, se formó en la comuna de Peñalolén el Movimiento de Pobladores en Lucha. Surgido desde la vida comunitaria, sus acciones no se insertan en las tradicionales demandas elevadas al Estado. La reivindicación y conquista de espacios de autonomía de los pobladores surgen, en este caso, en materia habitacional y urbana (Guzmán et al., 2009, p. 4). Esto ya marca una diferencia con respecto a las luchas por la vivienda desplegadas hasta ese momento, que habían incluido desde peticiones de vivienda al Estado hasta las tomas de terrenos masivas organizadas. Como afirman Sabatini y Wormald (2004, p. 80), los pobres urbanos que se movilizan hoy lo hacen más en función de su identidad como pobladores 
plenos, como propietarios inmobiliarios, que como pobres o desheredados. Ello no obsta para que las tradicionales demandas de acceso a una vivienda digna hayan continuado e incluso aumentado. Por ejemplo, en 2007 surgió en la comuna de La Pintana el Movimiento Pueblo Sin Techo, que tras una serie de movilizaciones reclamaba una mejora en las condiciones de acceso al Fondo Solidario de la Vivienda (Castillo, 2011, p. 136).

En 2010 se organizó la Federación Nacional de Pobladores (Fenapo), en la que participaban movimientos de allegados, deudores habitacionales, organizaciones de campamentos y familias damnificadas por el terremoto y maremoto de febrero de ese año, de alcance nacional. Esta catástrofe natural marca un antes y un después en la historia reciente del movimiento de pobladores, ya que supuso, con la constitución de la Fenapo, un intento de articulación y organización colectiva de comités de vivienda ya existentes, junto con la Asociación Nacional de Deudores Habitacionales (Andha), fundada pocos años antes (Angelcos, 2015, p. 10). Fueron, por tanto, tres los colectivos que participan en esta gran federación: los deudores habitacionales o pobladores "con techo", señalados por algunos autores como el gran problema social-habitacional del país (Rodríguez \& Sugranyes, 2005, p. 60); los colectivos de allegados, siendo el allegamiento un fenómeno que un año antes del terremoto, en 2009, afectaba a un 26\% de los hogares de la Región Metropolitana de Santiago (Congreso Nacional de Chile [CNC], 2012); y, finalmente, los afectados por el terremoto de 2010, que solo en Santiago de Chile colapsó más de 480 viviendas (La Tercera, 2010).

En 2011 se fundó el primer Comité de Vivienda Ukamau en la comuna de Estación Central, después de haber realizado sus iniciadores un diagnóstico sobre las necesidades básicas de las poblaciones. En ese trabajo constataron que una de las demandas más acuciantes de la población de la comuna -en especial la más pobreera el acceso a una vivienda en el mismo barrio en el que residían, con sus lazos familiares y sociales intactos (Entrevista a Aland Castro, vocero de Ukamau, 2016), de manera que desplegaron un repertorio de lucha renovado y en sintonía con el Movimiento de Pobladores en Lucha, que se plantea más adelante.

La amalgama de movimientos actualizó profundamente el repertorio de estrategias de contestación política en cuestiones de vivienda. Ello, según Tarrow (2011), fue una reacción a cambios en las oportunidades y restricciones a la acción, de carácter institucional, material o cultural, mientras la movilización era una respuesta defensiva al constreñimiento de oportunidades políticas (Cortés, 2014, p. 252). En este sentido, la llegada al poder de una coalición de gobierno de centro izquierda y el fracaso de las estrategias clásicas de las tomas en 2006, marca un hito para renovar profundamente la contestación política, con al menos tres innovaciones discursivas y prácticas importantes que destacan en los últimos ańos:

- La asunción del derecho a la ciudad en los discursos de los nuevos movimientos de pobladores, adaptado como derecho al "buen vivir" o a una "vida digna".

- El cambio de filosofía de contestación y de las estrategias de acción de los pobladores, desde la protesta al empoderamiento como agentes autogestionarios de sus propios proyectos de vivienda. 
- Intentos de institucionalización política mediante la concurrencia a las elecciones con marcas propias.

El concepto lefebvriano de 'derecho a la ciudad' es quizás la principal innovación conceptual introducida en los discursos de los movimientos de pobladores en los últimos años, un ejemplo de acción colectiva que se apropia y despliega un discurso asociado a este derecho de la ciudad, definido por la Carta Mundial por el Derecho a la Ciudad, que a su vez se inspira en la obra homónima de Lefebvre de 1968, como

el usufructo equitativo de las ciudades dentro de los principios de sustentabilidad, democracia, equidad y justicia social. Es un derecho colectivo de los habitantes de las ciudades, en especial de los grupos vulnerables y desfavorecidos, que les confiere legitimidad de acción y de organización, basado en sus usos y costumbres, con el objetivo de alcanzar el pleno ejercicio del derecho a la libre autodeterminación y un nivel de vida adecuado (Foro Social de las Américas [FSA], 2012, p. 184).

Para el poblador de hoy, su norte es el derecho a la ciudad; y la integración espacial y política con otros grupos sociales, un elemento crítico para tener éxito (Sabatini \& Wormald, 2004, p. 80). Los movimientos como el MPL o Ukamau adaptan este concepto al de "buen vivir" o "vida digna", ya que, según Harvey, (2012, p. xv), el propio concepto de derecho a la ciudad no deja de ser un "significante vacío" que ha de ser dotado de contenido por los movimientos sociales. Según el MPL, el objetivo de una vida digna "representa una lucha amplia que abarca distintas dimensiones del vivir y de habitar un territorio: la vivienda, la salud, la educación, el trabajo, nuestra identidad. En este camino, la casa propia es solo el principio de una larga y permanente lucha" (Guzmán et al., 2009, p. 10). El giro de diversos movimientos de pobladores hacia la conquista de un derecho a la ciudad constituye un proceso de empoderamiento y desalienación colectiva (Mathivet \& Pulgar, 2010). En este sentido, el lema "Nuestro sueño es más grande que la casa" que utiliza el MPL muestra que su lucha es más amplia y global que el acceso a un bien privado, apuntando a la voluntad de ser parte de la ciudad, de quedarse en el barrio, en la comuna de su elección; la voluntad de ser parte del proceso de toma de decisión, de tener un peso en las decisiones que tienen importancia en sus vidas (Mathivet \& Pulgar, 2010). Además, este lema es un homenaje a las luchas por la vivienda de los años sesenta lideradas por el Movimiento de Izquierda Revolucionaria (MIR), del cual se declaran herederos algunos nuevos movimientos de pobladores, como Ukamau (González, 2014).

En segundo lugar, destaca el empoderamiento de los movimientos de pobladores como agentes de cambio social en su propio espacio urbano. Como afirma Salazar (2012), "se trata de introducir en la práctica tradicional de la toma y compra de terrenos la autogestión de todo el proyecto de construcción de vivienda" (p. 191). Desde el punto de vista práctico, destaca la institucionalización de la autogestión inmobiliaria, es decir, la creación de inmobiliarias sociales y empresas constructoras de los pobladores que deciden actuar "por dentro y por fuera" de la institucionalidad vigente (Guzmán, 2009). Realizan así sus propios proyectos de vivienda, mediante la creación de instrumentos de autogestión para participar en 
los programas habitacionales estatales y, al mismo tiempo, llevar adelante procesos que apunten a una transformación integral de la construcción del hábitat popular (Castillo, 2011, p. 135). Se trata de un paso hacia el empoderamiento de los pobladores, de simples demandantes a sujetos activos en la producción de espacio en la ciudad de Santiago. Como afirma el mpl (2008): "Si este Estado no puede y los privados no quieren, seremos los pobladores los que construyamos nuestras propias soluciones". Otra estrategia interesante utilizada con el objetivo fundamental de que los pobladores puedan permanecer en la comuna en la que residen precariamente, consiste en realizar una labor de búsqueda de terrenos en la misma comuna. Más adelante, empiezan a cuestionar los instrumentos de planificación territorial, como los Planes Reguladores Comunales (PRC) y el Plan Regulador Metropolitano de Santiago (PRMs). Teniendo claro que el problema se centra en el acceso a suelo, exigen que estos instrumentos se modifiquen, en el sentido de garantizar una reserva de suelo destinado a la construcción de vivienda social (Castillo, 2011, p. 137). En este sentido, resulta fundamental la labor de nuevas entidades organizadas desde el MPL, como la Secretaría Popular de Planificación Territorial, que considera la puesta en marcha de propuestas alternativas para la planificación urbana, que contemplan todo el proceso de planificación y diseño comunitario de la población en que se va a vivir (Salazar, 2012, p. 191; Veloso, 2013).

Precisamente, esta estrategia ha conducido necesariamente a varios intentos de institucionalización política de diversos movimientos de pobladores: la "presión con toma" para forzar una concesión legalizable, tiende ahora a convertirse en "presión con propuesta" dentro de las instituciones políticas, para forzar cambios de legislación e incluso una nueva Constitución (Salazar, 2012, p. 206). El referente más evidente fue la formación del Partido Igualdad con miras a concurrir a las elecciones presidenciales de 2013. Fueron los movimientos de pobladores "Andha Chile a Luchar Democrático", "Movimiento del Pueblo Sin Techo" y "Movimiento de Pobladores en Lucha” los que consiguieron crear un partido de ámbito nacional para concurrir a las elecciones presidenciales y presentar, por primera vez en la historia del país, a una pobladora como candidata a la presidencia (Angelcos, 2015, p. 231). En este sentido, la participación de varios movimientos de pobladores en distintas elecciones busca presionar desde las instituciones para llevar a cabo una de las grandes reivindicaciones históricas de este movimiento: la aprobación de una ley del suelo y de la vivienda que se base en el "derecho de permanencia territorial de las familias" (Guzmán et al., 2009, p.13). Este proceso de institucionalización es la culminación del empoderamiento de los pobladores como actor político, superando así su tradicional dependencia respecto a actores exógenos (los partidos tradicionales de izquierda), relación basada en una lógica comunitaria defensiva provocada por la situación de exclusión social y política bajo la dictadura (Dubet, Tironi, Espinoza \& Valenzuela, 2016).

\section{Geografía de los conflictos de vivienda}

Las innovaciones y transformaciones en los discursos y estrategias de los movimientos de pobladores que se han mencionado son visibles en la geografía reciente de conflictos por la vivienda. La figura 1 recoge los principales conflictos territoriales por vivienda registrados en Santiago y su área metropolitana, en los últimos 
diez años; concretamente, desde el primer gobierno de Bachelet y la constitución del Movimiento de Pobladores en Lucha. Los conflictos por la vivienda recogidos aquí se refieren a confrontaciones entre movimientos de pobladores más o menos organizados y diversas administraciones del Estado (municipios, el Minvu, el Servicio Regional de Vivienda y Urbanismo [Serviu], otros ministerios, etcétera), en las que se utilizó un abanico variado de estrategias que muestran la intensidad de la confrontación, del conflicto. Tales estrategias han sido clasificadas en cuatro categorías:

- Participación formal. Participación siguiendo los cauces administrativos normales de consulta en un plan o proyecto que normalmente contempla la construcción o rehabilitación de viviendas.

- Participación activa. Presión más allá de los cauces habituales, pero sin grandes acciones de protesta: formación de comités de vivienda, asambleas de barrio, publicación de manifiestos o recogida de firmas.

- Protesta. Se trata de la acción clásica en cualquier conflicto territorial que consigue involucrar a bastantes ciudadanos: la protesta en la calle mediante manifestaciones regladas o autorizadas.

- Confrontación violenta o acciones ilegales. En esta categoría se agrupa el amplio abanico de acciones que van más allá del marco legal establecido de comportamiento en la vía pública. Se trata de acciones como las tomas de terrenos, las ocupaciones de edificios públicos, las acampadas en la vía pública o el hostigamiento a autoridades políticas y policía.

FIGURA I | Mapa de conflictos urbanos por la vivienda en el Área Metropolitana de Santiago 2006-2016

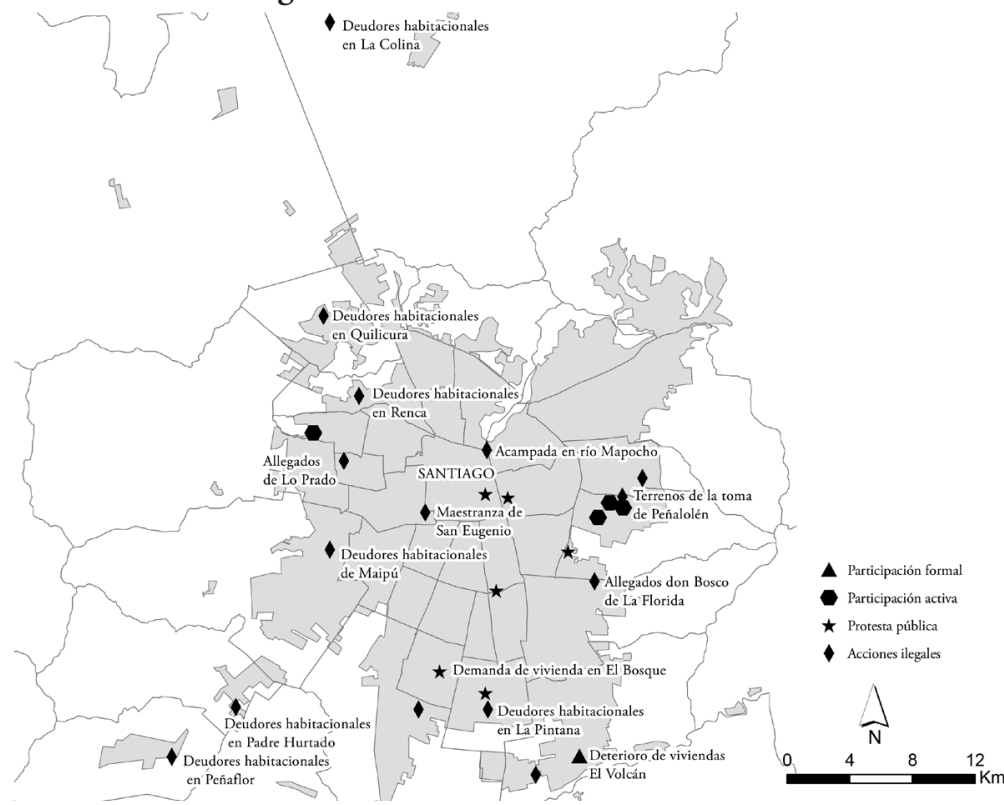

FUENTE ELABORACIÓN PROPIA 
El mapa anterior muestra 26 conflictos de vivienda. En primer lugar, llama la atención que en su mayor parte se localizan en áreas pericentrales de la metrópoli, con Peñalolén como la comuna que más conflictos por la vivienda ha registrado en la última década (cuatro). Prácticamente todas las comunas que rodean Santiago presentan algún caso de conflicto, desde Estación Central a Nuñoa, por San Joaquín o Providencia, cada una con un conflicto, respectivamente. Pese a que la comuna de Santiago es el centro urbano y posee una de las poblaciones más elevadas del área metropolitana, tan solo ha surgido allí un conflicto por la vivienda en los últimos años. Junto con esta ubicación pericentral, cabe mencionar también que el sur del área metropolitana concentra un buen número de conflictos por la vivienda, lo cual se explica por la importante concentración de poblaciones en diferentes situaciones de precariedad, así como por una gran cantidad de allegados y deudores habitacionales, descontentos con las políticas de vivienda.

Un segundo elemento que llama la atención es la variedad de actores que se involucran en conflictos relacionados con la vivienda, desde comités de barrio a asociaciones de escala nacional, lo cual muestra que los conflictos por la vivienda son un problema que dista mucho de estar resuelto; incluso, en años recientes, presenta varias escaladas de tensión, evidencia del dinamismo que aún conservan los movimientos de pobladores. En la amalgama de conflictos que se muestran en el mapa, un primer actor clave son los "pobladores con techo", agrupados en asociaciones de deudores habitacionales, así como la asociación "Andha Chile Democrático" (Asociación Nacional de Deudores y Deudores Habitacionales). En numerosas comunas han surgido conflictos vinculados a comités asociados de Andha, de pobladores "con techo", que protestan por las formas de gestionar el acceso a la vivienda. En concreto, protestan por las políticas de privatización de la deuda hipotecaria para los beneficiarios de subsidios públicos, así como por la asignación inadecuada o insuficiente de créditos hipotecarios y por los intereses que tienen que enfrentar, además de protestas por la mala calidad de las viviendas recibidas (sUR, 2011). Otro grupo de actores destacado es el de los movimientos de pobladores sin techo o allegados, que protestan por la demanda clásica a una vivienda digna en la misma comuna que residen. Reclaman mediante distintas estrategias el derecho a una vivienda, pero también el derecho a la ciudad, a una vida digna para el asentamiento en que habitan y contra las políticas de desplazamiento de familias a las periferias de la ciudad. En el apartado siguiente ello se analiza con más detalle.

Entre los elementos que más llaman la atención de esta cartografía de conflictos está el alto grado de confrontación que alcanzan, sobre todo si se comparan los conflictos en torno a la vivienda de ciudades como Santiago, con otras realidades geográficas latinoamericanas o europeas. En un 58\% de los conflictos estudiados, la presión de los pobladores se ejerció mediante acciones ilegales, especialmente ocupaciones de terrenos y corte de avenidas y calles; en pocos casos hubo enfrentamientos violentos con la policía. El marco institucional a partir del cual se despliega la política de vivienda y que dota de mecanismos de participación formal para el acceso a este bien o la expresión de inconformidades, incluidas las instancias jurídicas, parece completamente superado. El escenario mayoritario de comunicación entre los pobladores y el Estado chileno es la calle (si añadimos los conflictos expresados 
mediante manifestaciones, más de un $80 \%$ de ellos se expresa con estas estrategias). A continuación se analizará más detenidamente estas disfuncionalidades y complejidades, a partir de uno de los casos que han tenido más impacto social y mediático en los últimos años: el proyecto de vivienda en predios de la Maestranza de San Eugenio, liderado por el movimiento de pobladores Ukamau.

\section{La pugna por un lugar en la ciudad: el proyecto Maestranza de San Eugenio de Ukamau}

La Maestranza de San Eugenio, una de las áreas de talleres ferroviarios más importantes de Santiago, comenzó a ser construida en 1905, cuando la Empresa de los Ferrocarriles del Estado compró $147.762 \mathrm{~m}^{2}$ de terreno al sur de la Estación Alameda de Santiago (Ortega, 2013, p. 44). Junto a esta Maestranza y estación, comenzaron a surgir diversas industrias y talleres, y con ellos, los primeros barrios obreros, como la Población San Vicente, surgida en 1894, o la Población San Eugenio para ferroviarios, inaugurada en 1911 (Ibíd., p. 47). Durante décadas, la Maestranza de San Eugenio y sus industrias anexas constituyeron todo un símbolo de la industrialización del país, pero a comienzos de los años setenta, con la crisis del petróleo y la instauración del régimen militar, comenzó la decadencia del ferrocarril y, con ella, la de la propia Maestranza. Los planes del nuevo gobierno militar y su programa neoliberal se decantaron por desregular el transporte de pasajeros y por desmantelar la tupida red de ferrocarriles existente hasta esa época, en respuesta al déficit que arrastraba la compañía estatal (Thomson, 2012). Aunque los talleres ferroviarios siguieron funcionando, gran parte de la Maestranza y sus fábricas anexas fueron cerrándose, comenzando así un progresivo deterioro que dura hasta hoy. Algunos equipamientos importantes acabaron siendo derribados, como el histórico estadio Hugo Arqueros Rodríguez en el que jugaba el equipo de fútbol fundado por trabajadores de la Maestranza, que fue desmantelado en 2012. De esta manera, desde los años noventa quedó sin uso un inmenso conjunto de sitios de unas 35 hectáreas, en una posición central en la metrópoli (junto a las autopistas Central y del Sol, en el límite entre la comuna central de Santiago y la de Estación Central); por lo tanto, una localización muy interesante para el desarrollo inmobiliario.

En los alrededores del predio de EFE sí había gente y actividad; por ejemplo, la Población Nogales, constituida en los años sesenta por familias mayoritariamente inmigradas del campo y de bajos recursos. Se originó tras una toma de terrenos destinados a albergar a familias de las Fuerzas Armadas, toma que dio paso a un "campamento" que poco a poco fue tomando forma y siendo urbanizado por los propios vecinos. Hoy en día sigue siendo un asentamiento pobre, en una situación relativamente céntrica, pero con importantes problemas de allegamiento por la llegada de nuevos inmigrantes al barrio en los últimos ańos, y por el crecimiento natural de la población. En este contexto surgió el movimiento de pobladores Ukamau ("así somos", en aymara) en 1987, en el seno de la población, con el fin de ayudar a los jóvenes del barrio que vivían en un ambiente de marginalidad y en un barrio con un $30 \%$ de cesantía (entrevista a Aland Castro, 2016). Gracias al apoyo de una ONG, adquirieron unos terrenos en los que se edificó un centro social, que es hasta hoy la 
sede de la asociación. En los años noventa funcionó como un núcleo de resistencia y contestación política contra la nueva democracia, con una influencia importante del MIR, en el que se realizaban todo tipo de talleres y cursos para los niños de la población. Sin embargo, según señala Aland Castro (entrevista, 2016), "éramos los raros del barrio. Nos dábamos cuenta de que no teníamos comunicación con los vecinos del barrio; la mayoría eran ferianos que trabajan todo el día, algunos se desplazan como dos horas o más, cada día. Ellos no entendían de marxismo ni de discursos políticos”.

\section{Figura 2 | Plano de la Maestranza de San Eugenio}

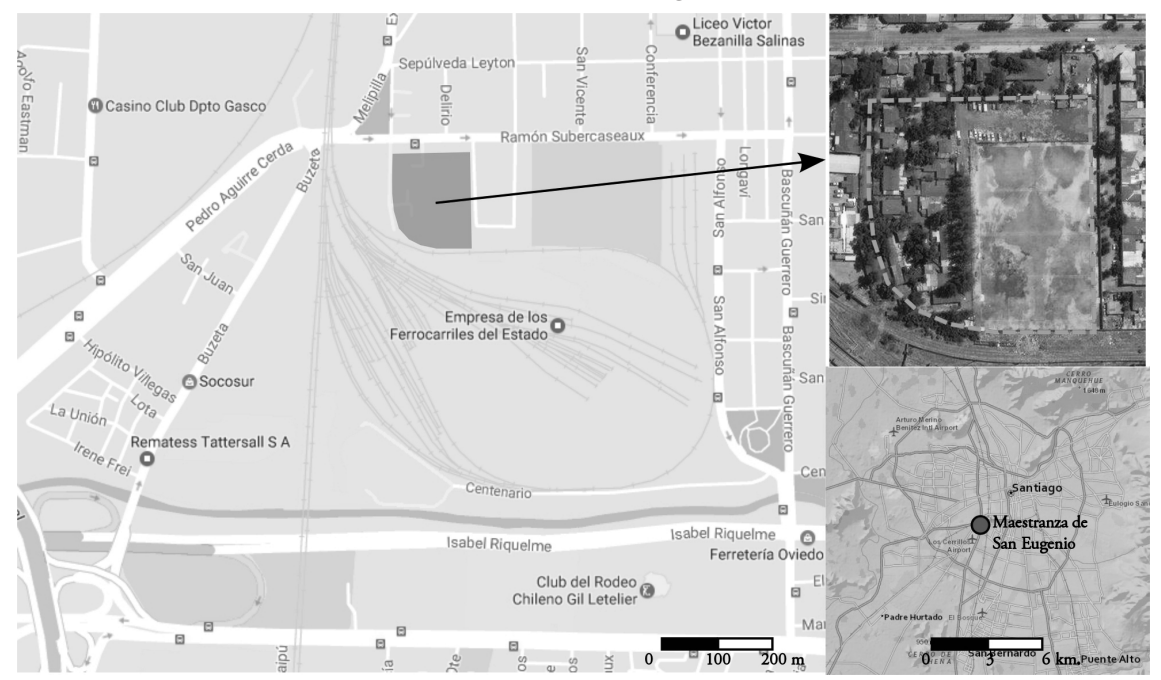

FUENTE ELABORACIÓN PROPIA

Por esta razón, en 2004 comenzaron a cambiar completamente de estrategia. A partir de un diagnóstico social en el barrio, concluyeron que el aspecto que más preocupaba a la población y por el que estaba dispuesta a movilizarse, era la vivienda:

Nos dimos cuenta de que la gente no se movilizaba (...) por la salud, quizás puntualmente, cuando uno está enfermo y no tiene para el médico, igual que con la educación. Con el trabajo tampoco. La gente no está dispuesta a jugarse su trabajo en una huelga, aunque sea para mejorar sus condiciones, pero con la casa es otra cosa. Una madre que ve que su hijo no puede jugar en la casa porque molesta, que no hay espacio apenas para ello (...) la demanda por una vivienda digna, eso es lo que moviliza al pueblo (entrevista a Aland Castro, 2016).

En 2011 fundaron el primer comité de vivienda, con el objetivo de "luchar por la vida buena”. Desde el inicio, se articuló como una organización cuya acción se dirigía a buscar soluciones habitacionales para los sin techo y los allegados del barrio, pero — como se ha expuesto - tenía como objetivo final la mejora de las condiciones de vida de toda la población, con acento especial en la educación y formación de los jóvenes. Una de las seńas de identidad de Ukamau es su insistencia 
en la necesidad de generar nuevas formas de conocimiento sobre la realidad. No se trata, sin embargo, de una preocupación exclusiva de este movimiento de pobladores; numerosos movimientos sociales en toda América Latina muestran un cuestionamiento cada vez más profundo de la modernidad capitalista y patriarcal, entendida como sistema complejo de dominaciones (antropocentrismo, capitalismo, patriarcado, eurocentrismo, colonialidad, etcétera) y construyen alternativas a las mismas, aunque sea de forma parcial y con limitaciones (Martínez, Casado \& Ibarra, 2012, pp. 20-21). En el caso de Ukamau, además de cursos y talleres sobre cuestiones ambientales y sociales, fundaron un equipo de fútbol para los niños del barrio, bautizado como "Gallo Rojo", en homenaje a una famosa canción antifranquista espańola.

La visibilidad y el conflicto de Ukamau comenzó con la constitución de una asamblea a la que fueron invitados todos los vecinos del barrio, independientemente de su ideología, con el fin de articular una demanda concreta. A partir de varias asambleas, decidieron actuar fundamentalmente con dos demandas: a escala nacional, la aprobación de una ley de vivienda y urbanismo que fuera más allá de los tiempos electorales, diera fin a las dinámicas de expulsión de la población pobre a la periferia y ordenara el caótico crecimiento urbano de comunas pericentrales como Estación Central, que disponen de instrumentos de planeamiento anticuados; a escala local, concibieron un gran proyecto de vivienda colectiva, autogestionado, en los terrenos de la Maestranza de San Eugenio, con el apoyo en el diseño del arquitecto Fernando Castillo Velasco, para 500 vecinos sin techo del barrio.

Su estrategia buscaba, ante todo, la atención mediática y política, para lo cual realizaron un abanico amplio de acciones de protesta: envío de cartas con su petición, manifestaciones, cortes de avenidas, protestas ante el Serviu, e incluso llegaron a ocupar el edificio de la Municipalidad de Estación Central, así como la propia estación. Su discurso se enmarcó en el derecho a la ciudad, entendido como el derecho a habitar en la misma comuna en la que nacieron, así como el derecho a una vida digna. En este sentido, consideraban su demanda de los terrenos públicos de la Maestranza como más legítima que la posibilidad de que fueran finalmente vendidos a la iniciativa privada para la construcción de barrios de rentas medias y altas, lo que contribuiría a la gentrificación del barrio.

Después de dos años de lucha y protesta, finalmente consiguieron el compromiso por parte de la Empresa de los Ferrocarriles del Estado (EFE) en cuanto a ceder una parte del predio de la Maestranza de San Eugenio para la construcción de 424 viviendas, según el proyecto presentado y defendido con Ukamau. La operación consistió en la compra, por parte del Serviu, de un terreno de unas dos hectáreas a EFE, con el fin de adjudicarlo para el proyecto de vivienda bautizado como "Ukamau", predio limitado por la propia Maestranza al sur, la calle Ramón Subercaseaux al norte, Santiago Watt al este y Exposición al oeste, y que ocupaba parcialmente una cancha de fútbol (figura 3). 
FIGURA 3 | Vista de algunas viviendas en el terreno comprado por Serviu para el proyecto de vivienda social Ukamau
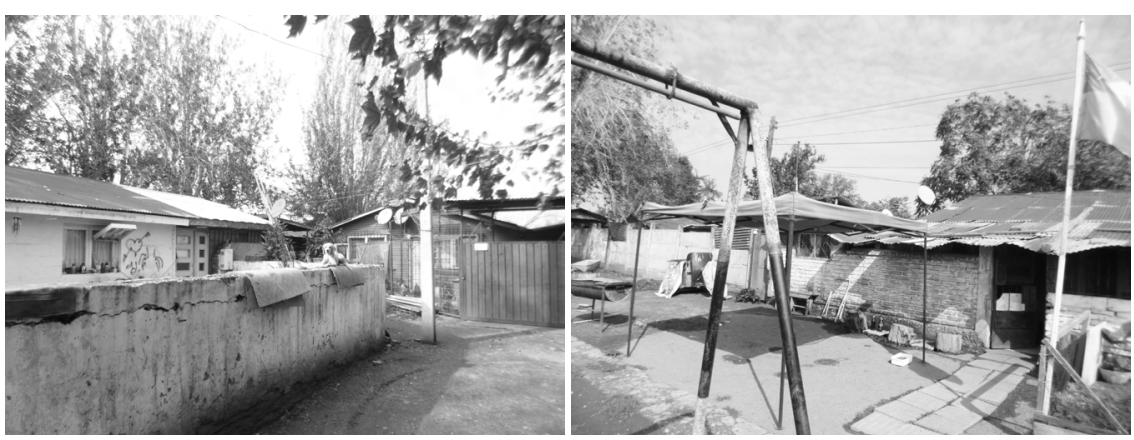

FUENTE ELABORACIÓN PROPIA

El conflicto de Ukamau por los terrenos de la Maestranza no es un conflicto finalizado, como la mayoría de los que se han dado en torno a la vivienda. En primer lugar, la Dirección de Obras Municipales comenzó a demorarse a la hora de otorgar permisos e iniciar el concurso público, y la construcción continúa sin comenzar. En segundo lugar, el terreno de dos hectáreas elegido presenta problemas de contaminación acústica, por la cercanía del tren y de varias vías con bastante tráfico, pero sin duda el problema más grave es que esos terrenos están ocupados por familias desde hace décadas. Parte de dichos predios fueron arrendados por la propia compañía EFE a familiares de trabajadores de los ferrocarriles, muchos de los cuales viven justamente en el terreno destinado a la construcción de 424 viviendas sociales, según el proyecto liderado por Ukamau. Como es de esperar, existe una fuerte oposición al traslado de esta población. Así lo expone Valeska, residente en Cancha no 2:

Hasta el 31 de diciembre pagábamos un arriendo a invia [Inmobiliaria Nueva Vía s.A., filial de la Empresa de los Ferrocarriles del Estado], pero la cuenta ya no existe. Acá vinieron hace no mucho unos topógrafos y unos obreros ahí a la cancha a hacer unas pruebas de suelo; entonces nos dijeron que el terreno se había vendido, pero aquí hay gente viviendo setenta años y nunca nos dijeron nada... Yo iba a hacer obra, a arreglarme el techo, ¿y ahora qué hago? Hay gente que arrendó habitaciones en septiembre y en diciembre [de 2015], se marcharon los inquilinos por los rumores del proyecto.

Un vendedor de fruta y verdura de la calle Exposición señala: "Es bien tranquilo acá, yo no cambio mi casa con mi patio y mi jardín por un departamento, aunque sea lujoso". Y una vecina de la misma calle comenta: “Acá son todo jubilados o trabajadores de EFE que llevan toda la vida aquí. Mi marido trabaja en EFE y seguramente nos trasladarán. Aunque lo intentamos, nunca nos dejaron comprar los terrenos”.

Con el proyecto de Ukamau se verían afectadas unas 23 familias que deberán abandonar sus casas, aunque según los vecinos son más. El conflicto podría derivar en una lucha de poder por el espacio urbano entre colectivos o clases distintas, 
pobladores sin techo versus trabajadores de EFE y sus familiares, más desmovilizados y menos organizados que los movimientos de pobladores (o mejor representados en sindicatos y partidos políticos tradicionales). Otra hipótesis podría ser la rearticulación del conflicto como una nueva protesta contra la gestión del proyecto de vivienda por parte del Serviu, que escoge en un terreno de 35 hectáreas las únicas dos parcelas donde hay vecinos viviendo desde hace décadas. Esta segunda opción requeriría de un desarrollo de alianzas multiclasistas, difícil de darse, sobre todo porque la compra de terrenos ya se realizó a finales de 2014, y el siguiente paso es dedicarlos a un proyecto de vivienda social, tal y como reclama Ukamau.

Quizás uno de los logros más significativos de Ukamau sea haber conseguido la integración de pobladores en un proyecto de transformación social, centrado en el acceso a una vivienda digna, en un contexto nacional de poblaciones con una importante desmovilización debido a problemas de delincuencia y precariedad laboral, y también al desarrollo de una cultura individualista (De la Jara, 2005). Sin embargo, su focalización hacia una propuesta concreta en unos terrenos determinados, que sustituye la tradicional estrategia de las tomas por la combinación de acciones de presión y negociación, hace que parte de su credibilidad y raison d'être dependa del éxito de su vía de actuación política-activista. Por otra parte, los vecinos residentes en los terrenos del proyecto Ukamau no poseen la larga tradición de movilización social de los pobladores, y prefieren canalizar el conflicto a través de las instituciones judiciales, interponiendo denuncias contra el procedimiento de compra de terrenos por parte del Serviu, por ejemplo, o reclamando a la propia administración un realojo justo. Ello podría conducir a una dilatación temporal importante del conflicto, difícil de asumir, especialmente para las 424 familias sin techo que necesitan una solución habitacional urgente, puesto que, como afirman en Ukamau, "tenemos 500 familias más esperando" (Entrevista a Aland Castro).

El conflicto, por lo tanto, va más allá de una simple pugna por el derecho a la vivienda. Muestra, por un lado, las enormes diferencias en el repertorio de protesta empleado por diferentes clases sociales en su disputa por un mismo espacio urbano; y por otro lado, la compra de esos terrenos parece una estrategia de desprestigio del movimiento de pobladores por parte de la administración. El discurso abanderado por Ukamau respecto del derecho a la ciudad y a una vida digna, materializado en un proyecto que implica el desahucio de familias, podría resultar cuestionable para muchos ciudadanos. Este conflicto muestra asimismo la dicotomía existente entre dos clases obreras: la clase obrera institucionalizada, sindicalizada y heredera de las luchas obreras de décadas anteriores y hoy profundamente desmovilizada, frente a otra clase obrera informal representada por los pobladores y por Ukamau, mucho menos institucionalizada y que busca nuevas formas de ciudadanía popular y su lugar en la ciudad.

Según como se desarrollen las negociaciones entre el Serviu, los vecinos del terreno y Ukamau, pueden crearse diferentes escenarios. En primer lugar, que las familias afectadas sean reubicadas por EFE, antigua propietaria de los terrenos, y la construcción autogestionada de un complejo de viviendas. En este caso, podrían darse idénticas demandas en otros terrenos de la Maestranza, que hoy también son estratégicos para desarrollar proyectos urbanísticos, lo que atentaría contra 
los intereses de constructoras que ya operan en la comuna de Estación Central levantando edificios de oficinas (figura 4). Un escenario diferente sería la reactivación del conflicto por parte de los vecinos afectados, seguramente a partir de su judicialización, estrategia poco empleada por el movimiento de pobladores. El principal argumento jurídico podría basarse en la ejecución improcedente de la compra de terrenos, sin negociación o consulta a los vecinos arrendatarios, algunos de ellos durante décadas. Ello podría suponer la congelación del proyecto y, como consecuencia, podría perjudicar los intereses de Ukamau, produciendo una desmovilización de pobladores, o desembocando en alguna acción violenta tendiente a la recuperación de terrenos como única estrategia efectiva para presionar a las administraciones. Conflictos como el de la Maestranza de San Eugenio muestran de manera clara cómo los que se desarrollan en torno a la vivienda en Santiago son de gran actualidad y complejidad, y representan una pugna entre clases sociales y un Estado que prefiere privilegiar exclusivamente los intereses del sector inmobiliario, lo cual explica la importante conflictividad territorial.

FIgura 4 Construcción de torres de oficinas de 30 pisos en las cercanías de la Maestranza de San Eugenio, sustituyendo la trama urbana tradicional de casas de clases obreras de una sola planta

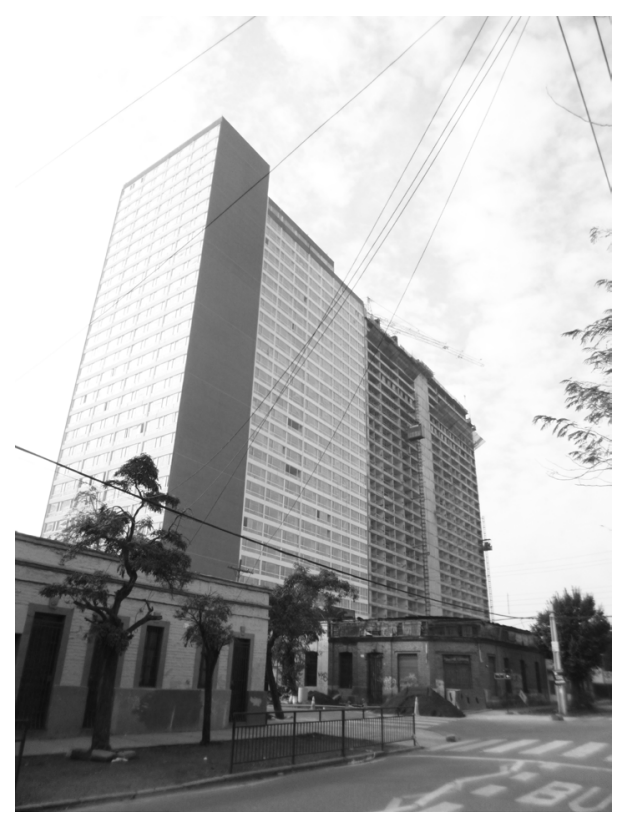

FUENTE ELABORACIÓN PROPIA 


\section{Conclusiones}

La atención académica prestada al movimiento de pobladores en los últimos años ha sido más bien limitada, aunque tanto la aparición de importantes novedades en las políticas públicas de vivienda como una transformación profunda de ese movimiento, merecen una mayor atención. La política urbana de corte neoliberal ha dejado una impronta profunda en Santiago, sobre todo desde el punto de vista social: se ha acentuado enormemente la segregación residencial, en parte al situar en las periferias los grandes proyectos de vivienda social. Frente a estas lógicas, el movimiento de pobladores protesta con discursos nuevos en torno a la vida digna o el derecho a la ciudad, y pasa de las tomas masivas a un abanico más amplio de estrategias. El resultado es una nueva geografía del conflicto urbano, con numerosos casos que surgen especialmente en la periferia inmediata de Santiago y con niveles de violencia notablemente altos.

En estos conflictos, las administraciones comunales parecen estar completamente alineadas con los intereses de las constructoras y empresas inmobiliarias. Ello se deduce, por una parte, de los numerosos casos de proyectos de vivienda social fallidos, que han dado lugar al fenómeno de los "pobladores con techo"; y por otra, del continuismo y persistencia de las políticas de subsidio a la vivienda, en lugar de la formulación de una política de Estado que acote el campo de acción de la iniciativa privada. En definitiva, tanto el paisaje de conflictos en torno a la vivienda, como los movimientos activos de pobladores, muestran hoy en día un gran dinamismo, que puede crecer notablemente en el caso de que proyectos alternativos, como el de Ukamau, vean finalmente su consecución.

Ahora bien, a los múltiples obstáculos puestos por diferentes administraciones, hay que ańadir la presencia de otro actor externo en pugna: la clase obrera de EFE, que puede acabar constituyendo un ejemplo de lucha de clases por el espacio urbano. De esta manera, el conflicto urbano por la vivienda requiere de nuevas miradas y aproximaciones multidisciplinares, que ayuden a desentrañar su complejidad y a poner en valor su potencia como motor de lucha por una ciudad más igualitaria y socialmente cohesionada.

\section{Agradecimientos}

Este trabajo es fruto de una colaboración entre el autor y el Centro de Estudios de Conflictos y Cohesión Social de Chile (coes).

\section{Referencias bibliográficas}

Angelcos, N. (2015). La construction du politique chez les nouvelles générations de pobladores au Chili. Tesis doctoral. París: École des Hautes Études en Sciences Sociales. 
Angelcos, N. \& Pérez, M. (2017). De la "desaparición" a la reemergencia: continuidades y rupturas del movimiento de pobladores en Chile. Latin American Research Review, 52(1), 94-109. https://larrlasa.org/articles/10.25222/larr.39/

Castells, M. (1973). Movimiento de pobladores y lucha de clases en Chile. EURE, 3(7), 9-35. http://eure.cl/index.php/eure/issue/view/20

Castillo, M. J. (2011). Producción y gestión habitacional de los pobladores. La autogestión de vivienda en Peñalolén y La Pintana, Santiago de Chile. Boletín CF+S 54. La ciudad a escala humana. XII Congreso Anual N-aerus, pp. 133-145. http://polired.upm.es/ index.php/boletincfs/article/view/2041

Biblioteca del Congreso Nacional de Chile (вСN). (2012). Reportes estadísticos comunales 2012. Índices de allegamiento. http://reportescomunales.bcn.cl/2012/index.php/Santiago

Cortés, A. (2014). El movimiento de pobladores chilenos y la población La Victoria: ejemplaridad, movimientos sociales y el derecho a la ciudad. EURE, 4O(119), 239260. http://dx.doi.org/10.4067/S0250-71612014000100011

De la Jara, A. M. (2005).El desafío de recuperar la vida social en condominios de vivienda social. En A. Rodríguez \& A. Sugranyes (coords.), Los con techo, un desafio para la politica de vivienda social. Santiago: Ediciones sur. http://www.sitiosur.cl/r.php?id=81

De Mattos, C.(2009). Santiago de Chile, globalización y expansión metropolitana; lo que existía sigue existiendo. En A. Rodríguez \& P. Rodríguez (eds.), Santiago, una ciudad neoliberal. Quito: Olacchi, 2009.

Del Romero, L. (2016). Conflicts in the city. Reflections on urban unrest. Nueva York: Nova Publishers.

Dubet, F., Tironi, E., Espinoza, V. \& Valenzuela, E. (2016). Pobladores. Luchas sociales y democracia en Chile. Santiago de Chile: EdicionesUniversidad Alberto Hurtado.

Ducci, M. E. (1997). Chile: el lado oscuro de una política de vivienda exitosa. EURE, 23(69), 99-115. http://www.eure.cl/index.php/eure/article/view/1164

Foro Social de las Américas (2004). Carta Mundial por el Derecho a la Ciudad. Revista Paz y Conflictos, (5), 184-196. http://www.ugr.es/ revpaz/documentacion/rpc_n5_2012_ doc1.pdf

Francés, V. (2016). Chile recupera. Recuperando relatos sobre problemáticas y luchas por la vivienda y la ciudad en Santiago de Chile. Red Contested Cities. chilerecupera.net

González, D. (2014). Vigencia del proyecto político de Miguel Enríquez. Punto Final, (815), 17 de octubre de 2014. http://www.puntofinal.cl/850/ukamau.php.

Guzmán, R., Renna, H., Sandoval, A. \& Silva, C. (2009). Movimiento de pobladores en lucha: "A tomarse Peñalolén para conquistar la ciudad". Cuadernos sur. Santiago: Ediciones sur. http://www.sitiosur.cl/publicacionescatalogodetalle.php?PID=3539\#descargar

Harvey, D. (2012). Rebel cities: from the right to the city to the urban revolution. Nueva York: Verso.

Klein, N.(2007). The shock doctrine. The rise of disaster capitalism. Nueva York: Henry Holt and Company.

latercera.com (2010, 5 de marzo). Nacional: Minvu: 480 edificaciones fueron declaradas inhabitables en la Región Metropolitana. http://archive.is/9bZK

Martínez, Z., Casado, B. \& Ibarra, P. (2012). Movimientos sociales y procesos emancipadores. Cuadernos de Trabajo (57) [Hegoa. Instituto de Estudios sobre Desarrollo y Cooperación Internacional]. http://www.ehu.eus/ojs/index.php/hegoa/article/view/10309/9501 
Mathivet, C. \& Pulgar, C. (2010). El Movimiento de Pobladores en Lucha, Santiago, Chile. En Diálogos, propuestas, historias para una Ciudadanía Mundial, Dossier 2011-2012. http://base.d-p-h.info/es/fiches/dph/fiche-dph-8502.html

Movimiento de Pobladores en Lucha(2008). Comunicado nº 9, 18 de julio de 2008. http:// mplchile.blogspot.cl/

Ortega, A. (2013). Rieles que unen. Patrimonio ferroviario y configuración del paisaje cultural urbano del ferrocarril al sur entre Estación Central y San Bernardo (1905-2013). Tesis de Maestría, Instituto de Estudios Urbanos y Territoriales. Santiago: Pontificia Universidad Católica de Chile. http://estudiosurbanos.uc.cl/images/tesis/2013/ MDU_Andrea_Ortega.pdf

Oxhorn, P. (2004). La paradoja del gobierno autoritario: organización de los sectores populares en los ochenta y promesa de inclusión. Política (43), 57-83.

Peck, J., Theodore, N. \& Brenner, N. (2013). Neoliberal urbanism redux? International Journal of Urban and Regional Research, 37(3), 1091-1099. http://dx.doi.org/10.1111/14682427.12066

Rodríguez, A. \& Sugranyes, A. (2005). El problema de vivienda de los "con techo". En A. Rodríguez \& A. Sugranyes (coords.), Los con techo, un desafio para la política de vivienda social (pp. 59-78). Santiago: Ediciones sur. http://www.sitiosur.cl/r.php?id=81

Rodríguez, A. \& Sugranyes, A. (2009). El traje nuevo del emperador: las políticas de financiamiento de vivienda social en Santiago de Chile. En A. Rodríguez \& P. Rodríguez (eds.), Santiago, una ciudad neoliberal. Quito: Organización Latinoamericana y del Caribe de Centros Históricos (Olacchi). http://www.sitiosur.cl/tp.php?pid=73

Sabatini, F. \& Wormald, G. (2004). La guerra de la basura de Santiago: desde el derecho a la vivienda al derecho a la ciudad. EURE, 30(91), 67-86. http://dx.doi.org/10.4067/ S0250-71612004009100005

Salazar, G. (2012). Movimientos sociales en Chile. Santiago: Uqbar Editores.

sur (2011). Mapa de conflictos urbanos en Santiago. http://mapadeconflictos.sitiosur.cl/ index 1 .php?tipo $=4 \&$ searchInput $=$

Tarrow, S. (2011). Power in movement. Social movements and contentious politics. Cambridge: University of Cambrige.

Thomson, I. (2012). Los ramales ferroviarios de Chile: auge y agonía. En Amigos del tren. http://amigosdeltren.cl/ramales-ferroviarios-auge-y-agonia

Trivelli, P. (2009). Sobre la evolución de la política urbana y la política de suelo en el Gran Santiago en el periodo 1979-2008. En A. Rodríguez \& P. Rodríguez (eds.), Santiago, una ciudad neoliberal. Quito: Organización Latinoamericana y del Caribe de Centros Históricos (Olacchi). http://www.sitiosur.cl/tp.php?pid=73

Yates, J. S. \& Bakker, K. (2014). Debating the "post-neoliberal turn" in Latin America. Progress in Human Geography, 38(1), 62-90. http://dx.doi.org/10.1177/0309132513500372

Zibechi, R.(2008). Ecos del subsuelo: resistencia y política desde el sótano. En A. Ceceña (coord.), De los saberes de la emancipación y de la dominación (pp. 71-99). Buenos Aires: Consejo Latinoamericano de Ciencias Sociales (Clacso). http://biblioteca.clacso.edu. ar/ar/libros/grupos/cecen/06zibe.pdf 